\title{
A case report- tuberculous dislocation of pediatric hip managed by naseer awais external fixator
}

\begin{abstract}
We describe a technique of 'cross-hip distraction' to reduce a dislocated hip, with subsequent treatment of chronic granulomatous (Tuberculosis) infection. A 10 years old boy presented with a tubercular dislocation hip and collapsed femoral head. Examination revealed a leg length discrepancy of $7 \mathrm{~cm}$ and irritable hip, with chronic discharging sinus. A staged technique was used for infection clearance by antituberculous therapy at the same time ( 2 weeks after start ATT) gradually relocated the hip by distraction with a cross-hip mono lateral external fixator; we used Naseer Awais external fixator (Locally made of stain less steel with one plane and one threaded rod) to relocate the hip and establish normal anatomy. A good functional outcome was achieved with a minimal invasive procedure.
\end{abstract}

Keywords: External fixator, Arthritis, Limb length discrepancy, Sequestectomy
Volume 9 Issue 2 - 2017

\section{SM Rabiul Islam}

Department of Orthopaedic Surgery, Raja Isteri pengiran Anak saleha (RIPAS) Hospital, Brunei Darussalam

\author{
Correspondence: SM Rabiul Islam FICS, Department of \\ Orthopaedic Surgery, Raja Isteri pengiran Anak saleha (RIPAS) \\ Hospital, Bandar Seri Begawan, Brunei Darussalam, \\ Email rabieshan@gmail.com
}

Received: August 29, 2017 | Published: October 12, 2017

\section{Introduction}

Tuberculous arthritis results from mycobacterial invasion of a joint space, which can occur through Haematogenous spread, direct inoculation from trauma or surgery, Contiguous spread from an adjacent site of osteomyelitis or cellulitis and Lympatogenous spread. Common affected group are low socioeconomic and immunocompromised patient. Despite in-depth research into the pathophysiology and treatment of tubercular arthritis, the morbidity and mortality are still significant. Diseases such as cancer, diabetes, alcoholism, cirrhosis, and uraemia increase the risk for chronic granulomatous infection. Usually, predisposing conditions are associated with particular types of causative organisms. Tubercular arthritis difficult to treat is not uncommon, incidence was reported to be 2.5 per $1,00,000 .{ }^{1}$ Tuberculous septic arthritis with osteomyelitis may result in bone destruction, avascular necrosis, dislocation, growth abnormalities and damage to articular cartilage. ${ }^{1-3}$ With the development of antimicrobial agent mortality has been dramatically reduced with long-term sequelae occurring in only $5 \%{ }^{2}$ Antitubercular agent is the mainstay of treatment of tubercular arthritis and osteomyelitis which requires a staged approach with initial clearance followed by reconstruction.

We present a case report about the management of a complex reduction of the hip involving extensive femoral osteomyelitis, a dislocated hip and avascular necrosis/collapse of the femoral head following tubercolous septic arthritis in a 10-year-old boy. A staged approach was used with clearance of femoral osteomyelitis, cross-hip distraction. ${ }^{4}$

\section{Case report}

A ten years old boy presented to Orthopaedic outpatient department, Mayo hospital, Lahore, with the complaints of limping gait, chronic discharging sinus at left hips for 9 months. Nine months ago patient's had a history of fall from tree about 6 feet height, sustained injury to his left hip area, after that his left hip developed pain swelling restricted movement unable to bear weight on his left lower limb.

Patient was from a low socioeconomic family, normal birth history, previously healthy, Immunized, having two sisters three bothers all were healthy He had no other medical problem.

On general physical examination Patient was below average weight and height, well oriented time space and person.Haemodynamically stable. No abnormality was detected on systemic examination. On local examination there was a discharging sinus lateral aspect of Left upper thigh, Left hip was flexed, adducted and internally rotated. Trochanter was migrated proximally; Left lower limb was $7 \mathrm{~cm}$ short. Distal neurovascular status was intact.

Total leucocytes count (TLC) was normal, Lymphocyte count increased, high erythrocyte sedimentation rate (ESR), Complement reactive protein (CRP) was positive. Biopsy and Culture sensitivity revealed chronic granulomatous disease. So our diagnosis was' tubercular arthritis of Left hip joint with neglected posterior dislocation and Chronic Osteomyelitis of Left femur.

We started treatment with Anti Tubercular Therapy (ATT) for 12 months with Streptomycine $-500 \mathrm{mg}$ intramuscularly once daily for 2 months, Refampicin $300 \mathrm{mg}$ once daily for 12 months, Isonizid $150 \mathrm{mg}$ once daily for 12 months and pyrazynamide 750 $\mathrm{mg}$ once daily for 2 months. Ethambutol was not given as it is not recommended in children because of its adverse effect on optic nerve. Infection was improved gradually with subsiding of the discharging sinus, improvement of the general condition and normalization of CRP and ESR. Surgical treatment was started at same time with the medical treatment. Sequestectomy \& curettage. Across hip Naseer Awais External fixator (NA Ex fix, ) to bring the proximally migrated femoral head in acetabular cavity (anatomical position) to overcome limb length discrepancy which took total 35 days $2 \mathrm{~mm}$ per day. Once femoral head came to the position NA Ex Fix was removed and Hip Spica cast was applied for immobilization for 10 weeks. Hip spica cast was removed after 10 weeks. Physiotherapy was started, and gradual weight bearing walk was allowed. On post-operative follow up evaluation Patient was able to stand and walk unsupported without limping, was able to sit in squatting position painless.Figures 1-11

\section{Discussion}

Condition to treat the principles of treatment of tubercolous septic arthritis of hip joint is based upon the stage of the disease, but in the presence of a dislocated hip and an absent femoral head one faces a formidable task. Traditionally, in the presence of osteomyelitis of the femur and dislocation, excision arthroplasty would have been the first choice in adults. Although the Girdle stone procedure ${ }^{6,7}$ may reduce pain, the functional outcome is often poor. Studies of the conversion of a Girdle stone pseud arthrosis to an arthroplasty have 
shown improvements in the Harris hip score 8 personal satisfaction and activities of daily living, but only in the absence of infection..$^{8-10}$ Even in the case Extensive femoral chronic osteomyelitis and septic/ Tubercular arthritis hip is a difficult of infected total hip arthroplasties, two-stage revision has achieved a 92\% infection-free rate with improvement in the Harris hip score. ${ }^{11}$

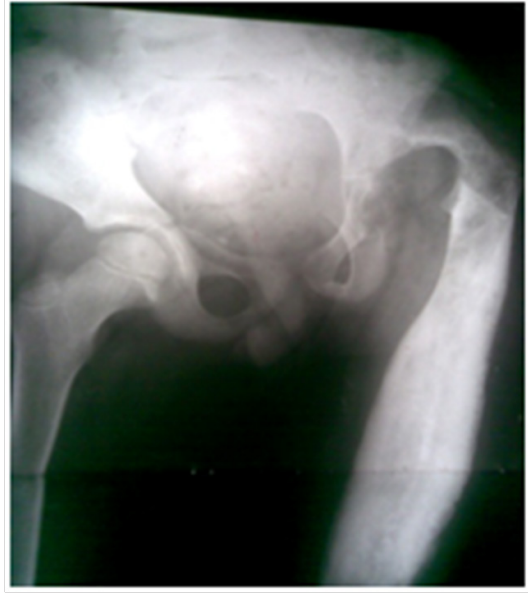

Figure I Initial radio graph- Femoral head dislocated supra-laterally, Shaper cook deformity of left femoral cortices are sclerosed, a large hyper dense area in the medullary cavity sequestrum. Pelvis tilted on the right.

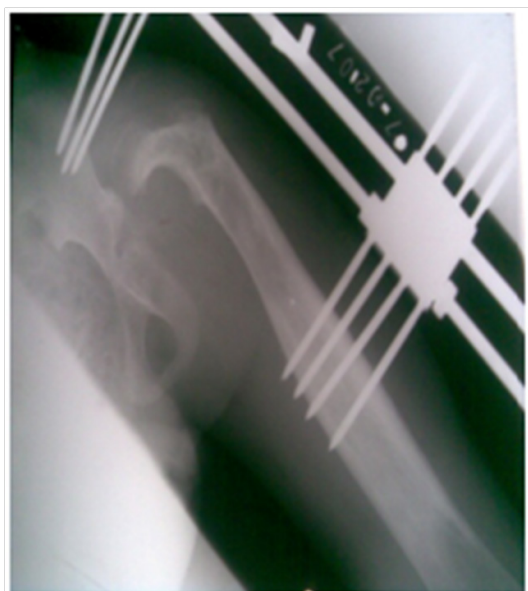

Figure 2 Across hip Naseer Awais External Fixator applied for control distraction of proximally migrated femoral head, $2 \mathrm{~mm}$ per day.

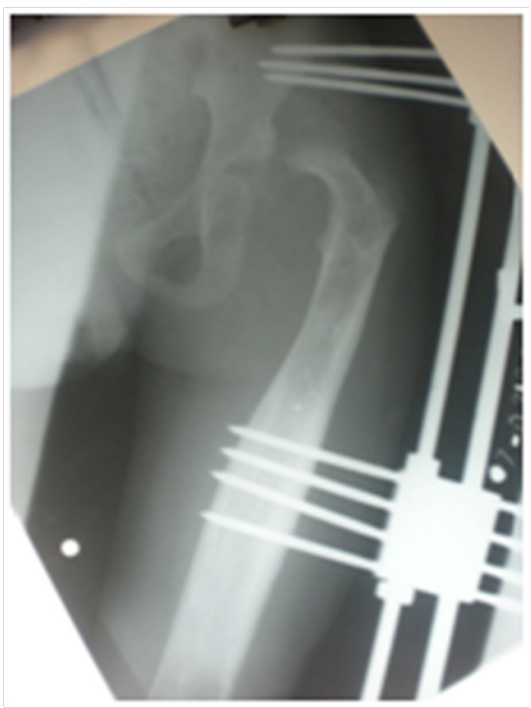

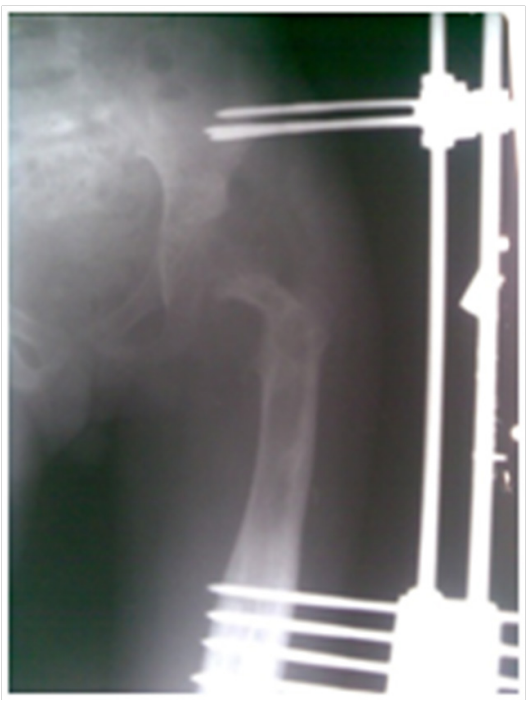

Figure 3\&4 Showing distraction started process is going on $2 \mathrm{~mm}$ per day, to avoid Neuro-vascular injury.

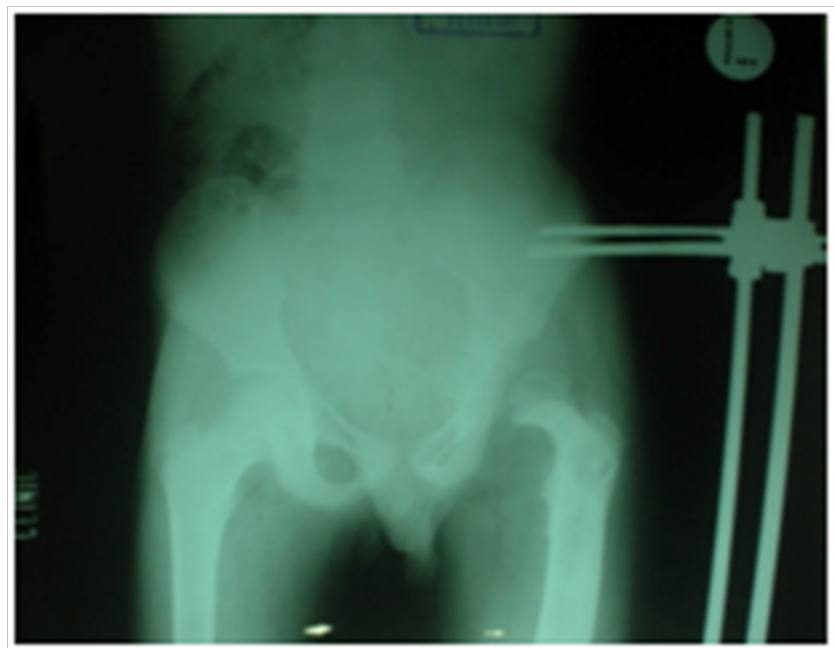

Figure 5 showing Radiograph 3 weeks after distraction is on progress by applying NA Ex Fix.

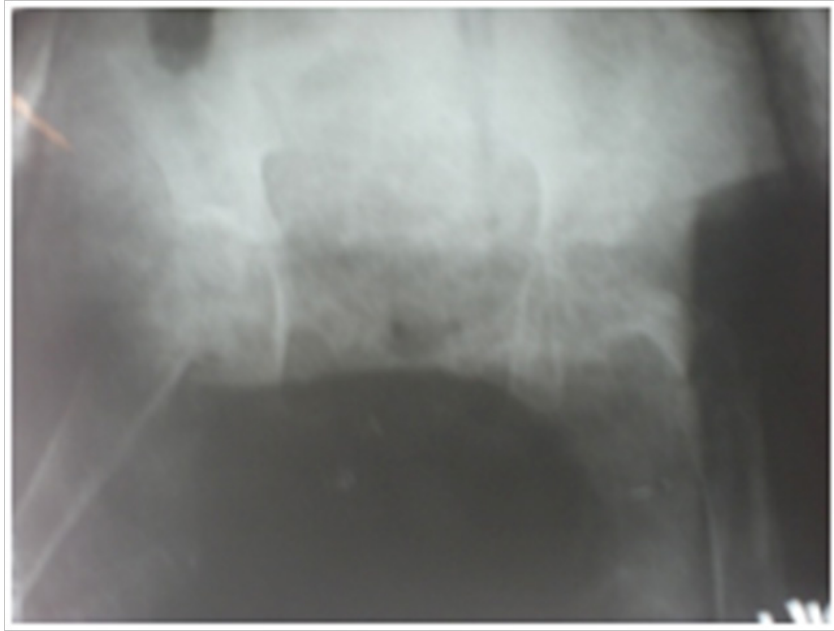

Figure 6 After removal of ex fix and application of Hip Spica cast. 


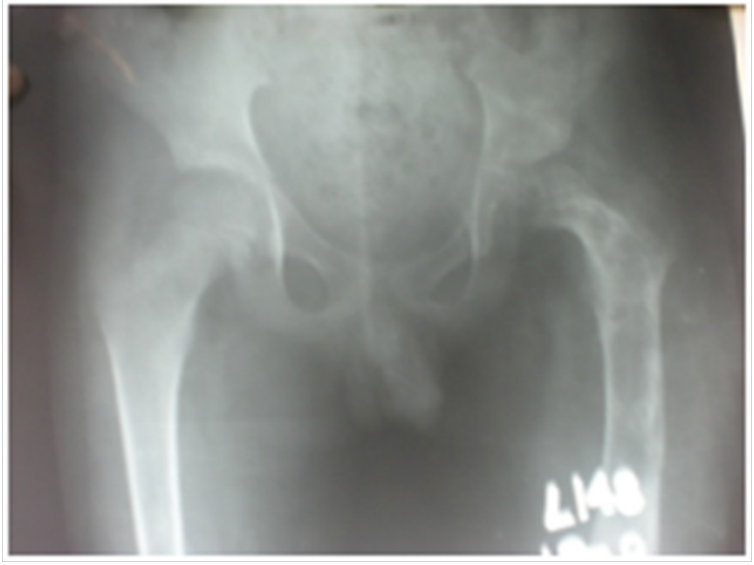

Figure 7 After completion of treatment and removal of hip Spica cast.
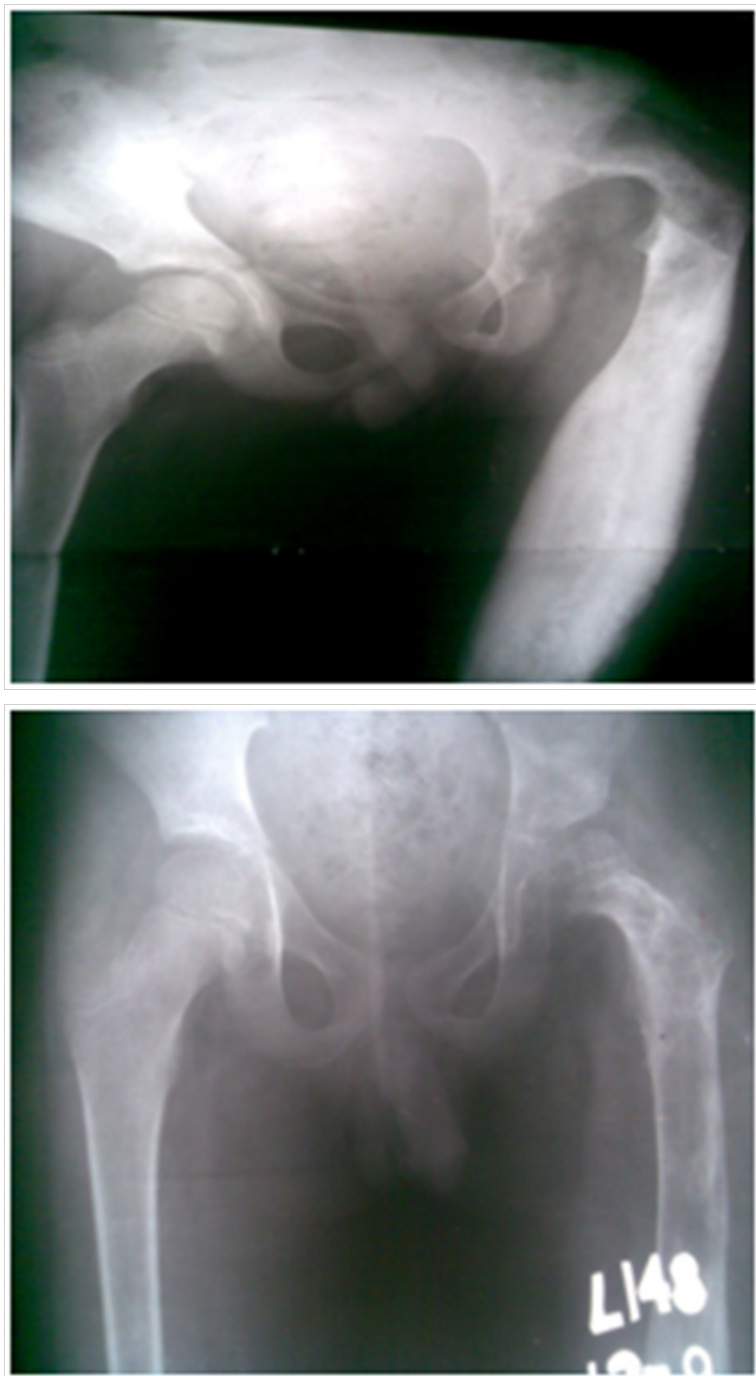

Figure 8\&9 Compare initial Radiograph and after completion of treatment.

Grill described cross-hip distraction in six hip dislocations diagnosed late. ${ }^{12}$ A Wagner fixator (Synthesis, Monument, and Colorado) was used after extensive muscle release to distract the hip followed by open reduction and pelvic osteotomy. Complications included femoral neck fracture in one and Varus bowing of the femoral neck in two. This technique has also been used in the treatment of avascular necrosis of the femoral head in children ${ }^{13}$ and central fracture- dislocation of hip ${ }^{14}$ with a good outcome. Distraction with a Kalnberz external fixator ${ }^{15}$ and marginal osteotomy has been used in the management of the sequelae of a septic hip. His outcome was a stable hip but at the expense of limitation of movement. Lai Liu \& $\mathrm{Liu}^{16}$ have shown good results with Wagner tibiofemoral distraction followed by total hip replacement in ${ }^{17}$ congenitally dislocated hips. Irreducibility, over shortening, nerve palsy, dislocation and femoral fractures were avoided. Leg length was regained with an improved Harris hip score. Manzotti et al. ${ }^{18}$ described hip reconstruction and lengthening in ${ }^{15}$ patients using a hybrid Ilizarov method for late sequelae of septic arthritis of hip. We use Naseer Awais External fixator is a simple unidirectional uniplanar fixator easy to handle by the patient and attendant for distraction. We did simply distraction followed by hip Spica cast without doing any osteotomy or internal fixation. The mechanical axis can be corrected with proximal femoral osteotomy and lengthening through a distal osteotomy with satisfactory results although a late total hip replacement may be difficult. Treatment of the chronically dislocated hip is controversial with most studies focusing on congenital dislocation and dysplastic hips. Femoral shortening, either by metaphyseal resection or subtrochanteric osteotomy, trochanteric osteotomy with distal advancement and soft-tissue release has been advocated. ${ }^{17,19,20}$ These procedures are technically demanding and need careful study of the anatomy. A wide range of prostheses has been advocated. Dislocation and nerve palsy are the most common complications followed by failure of the implant, nonunion of the osteotomy site and peri-prosthetic fracture. The reported incidence of nerve injury is in the range of $5 \%$ to $6 \%$ for an acute correction. A large intra-operative blood loss has been documented with a total post-operative complication rate of $33 \%$.

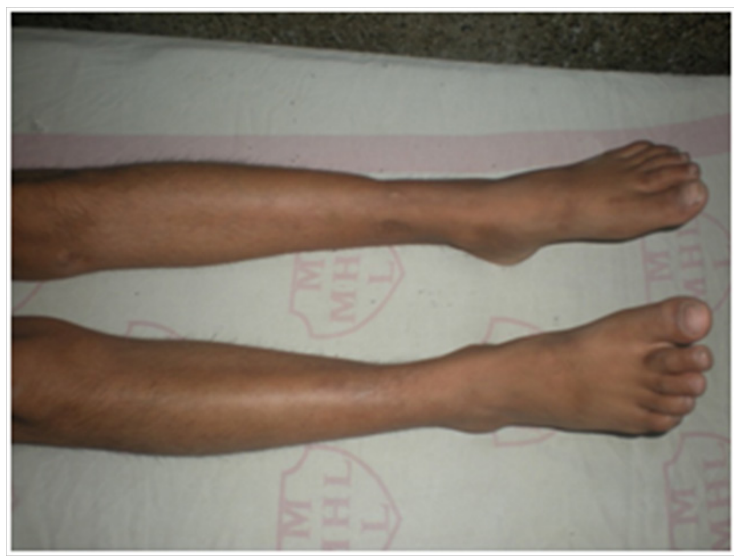

Figure 10 (Lt) Both limbs are equal length after treatment.

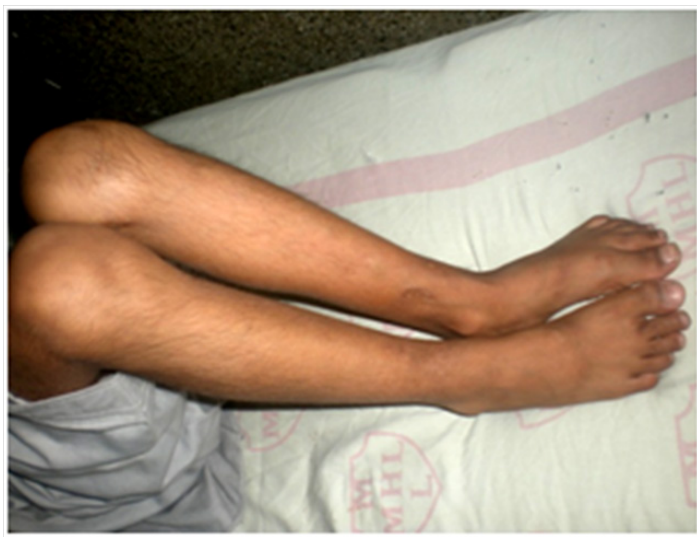

Figure I I (Rt) Galeazzi test negative. 


\section{Conclusion}

Tubercular dislocation of hip are common among pediatric patients, it is difficult to treat the infection as well as dislocated joint. Open reduction internal fixation is an extensive invasive procedure, which will cause further complication. It will increased patient's morbidity motility rate, increase treatment cost and prolong hospital stay. We managed such a case successfully by minimal invasive technique, applying Naseer Awais External Fixator, with out making any surgical wound or massive invasion; we did under Image intensifier guidance, with low cost and short hospital stay and minimal invasive technique with good functional outcome.

\section{Acknowledgements}

None.

\section{Conflicts of interest}

The authors declare no conflict of interest.

\section{References}

1. Peltola H, Vahvanen V. A comparative study of osteomyelitis and purulent arthritis with special reference to aetiology and recovery. Infection. 1984;12(2):75-79.

2. Petersen S, Knudsen FU, Andersen EA, et al. Acute haematogenous osteomyelitis and septic arthritis in childhood: a 10-year review and follow-up. Acta Orthopaedica Scand. 1980;51:451-457.

3. Peters $\mathrm{W}$, Irving $\mathrm{J}$, Letts $\mathrm{M}$ Long-term effects of neonatal bone and joint infection on adjacent growth plates. $J$ Paediatr Orthop. $1992 ; 12: 806-810$.

4. Cierny G, Mader JT, Penninck JJ. A clinical staging system for adult osteomyelitis. Clin Orthop. 2003;414:7-23.

5. Choi H, Pizzutillo PD, Bowen R, et al. Sequelae and reconstruction after septic arthritis of the hip in infants. $J$ Bone Joint Surg [Am]. 1990;72(8):1150-1165.

6. Bourne RB, Hunter GA, Rorabeck CH, et al. A six-year follow-up of infected total hip replacement managed by Girdle stone arthroplasty. $J$ Bone Joint Surg [Br]. 1984;66(3):340-3543.

7. McElwaine JP, Colville J. Excision arthroplasty for infected total hip replacements. J Bone Joint Surg Br. 1984;66(2):168-171.
8. Harris WH. Traumatic arthritis of the hip after dislocation and acetabular fractures: treatment by mold arthroplasty. $J$ Bone Joint Surg [Am]. 1969;51:737-755.

9. Charlton WPH, Hozack WJ, Teloken MA, et al. Complications associated with reimplantation after Girdlestone arthroplasty. Clin Orthop. 2003;407:119-126.

10. Schroder J, Saris D, Besselaar PP, et al. Comparison of the results of the Girdlestone pseudarthrosis with reimplantation of a total hip replacement. Int Othop. 1998;22(4):215-218.

11. Haddad FS, Muirhead-Allwood SK, Maktelow ARJ, et al. Two-stage uncemented revision hip arthroplasty for infection. $J$ Bone Joint Surg Br. 2000;82(5):689-694.

12. Grill F. Correction of complicated extremity deformities by external fixation. Clin Orthop. 1989;241:166-176.

13. Kucukkaya M, Kybukcuoglu Y, Ozturk I, et al. Avascular necrosis of the femoral head in childhood: the results of treatment with articulated distraction method. J Paediatr Orthop. 2000;20(6):722-728.

14. Vaatainen U, Makela A. Treatment of a central fracture-dislocation of the hip using external fixation with iliofemoral distraction. $J$ Orthop Trauma. 1993; 7:521-524.

15. Krumins M, Kalnins J, Lacis G. Reconstruction of the proximal end of the femur after haematogenous osteomyelitis. J Paediatr Orthop. 1993;13(1):63-67.

16. Lai K, Liu J, Liu T. Use of iliofemoral distraction in reducing high congenital dislocation of the hip before total hip arthroplasty. $J$ Arthroplasty. 1996;11(5):588-593.

17. Paavilainen T, Hoikka V, Solonen KA. Cementless total replacement for severelydysplastic or dislocated hips. J Bone Joint Surg Br. 1990;72(2):205-211.

18. Manzotti A, Rovetta L, Pullen C, et al. Treatment of the late sequelae of septic arthritis of the hip. Clin Orthop. 2003;410:203-212.

19. Garvin KL, Bowen MK, Salvati EA, et al. Long-term results of total hip arthroplasty in congenital dislocation and dysplasia of the hip. $J$ Bone Joint Surg Am. 1991;73(9):1348-1354.

20. Ito $\mathrm{H}$, Matsuno $\mathrm{T}$, Minami $\mathrm{A}$, et al. Intermediate-term results after hybrid total hip arthroplasty for the reatment of dysplastic hips. $J$ Bone Joint Surg Am. 2003;85(9):1725-1732. 